\title{
Secondary fatty alcohols of Mycobacterium xenopi
}

\author{
SRINIVAS Alugupalli and LeNNART LARSSON*
}

Department of Medical Microbiology, University of Lund, Sölvegatan 23, 22362 Lund, Sweden

(Received 20 July 1992; accepted 26 August 1992)

\begin{abstract}
Secondary alcohols of Mycobacterium xenopi were studied by gas chromatography and gas chromatography-mass spectrometry. Mycobacterial cells were hydrolysed and the liberated alcohols separated by extraction and analysed both underivatized and as trimethylsilyl-, methyl ether- and pentafluorobenzoyl derivatives. Seven straight-chain secondary alcohols containing from 18 to 24 carbon atoms and two branched-chain secondary alcohols with 21 and 23 carbon atoms were present in all of the studied strains.
\end{abstract}

\section{Introduction}

Mycobacteria and related organisms characteristically contain high-molecular-mass 3-hydroxy-2-alkyl branched fatty acids known as mycolic acids (Minnikin, 1982). Wax-ester mycolates have been found in only a limited number of mycobacterial species, among them the Mycobacterium avium complex, M. terrae, M. phlei and $M$. xenopi (Minnikin et al., 1985). These mycolates are readily hydrolysed to yield $\omega$-carboxy mycolates and free secondary alcohols (Jimenez \& Larsson, 1986); the latter are useful chemical markers in species identification of mycobacteria by gas chromatography (GC) and gas chromatography-mass spectrometry (GC-MS) (Jantzen et al., 1989; Jimenez \& Larsson, 1986).

The most commonly found secondary alcohols in mycobacteria that contain wax-ester mycolates are 2octadecanol and 2-eicosanol (Luquin et al., 1991). In addition, 2-docosanol has also been demonstrated in $M$. xenopi and $M$. phlei (Larsson et al., 1989; Luquin et al., 1989; Toriyama et al., 1982). Recently, we detected 2nonadecanol in strains of the $\boldsymbol{M}$. avium complex after extracting alcohols from whole-cell hydrolysates and identification by GC-MS (Alugupalli et al., 1992). In the present study, the same methodology was used for detailed analysis of the secondary alcohols of $M$. xenopi.

* Author for correspondence. Tel 46-46173289; fax 46-46189117.

Abbreviations: EI, electron impact, NICI, negative ion-chemical ionization; BSTFA, N,O-bis(trimethylsilyl)trifluoroacetamide; METH, methyl ether; PFBO, pentafluorobenzoyl; TMS, trimethylsilyl.

\section{Methods}

Chemicals. Pentafluorobenzoyl chloride (PFBO-Cl) was purchased from Janssen; $N, O$-bis(trimethylsilyl)trifluoroacetamide (BSTFA), silver oxide and methyl iodide from Fluka; pyridine from Merck; triethylamine from Sigma; and the solvents acetonitrile and hexane from Lab Scan. All the chemicals were of analytical grade.

Micro-organisms. Fifteen clinical isolates of $M$. xenopi and one reference strain (NCTC 10042) were examined. The mycobacteria were identified by standard biochemical tests. The bacteria were grown in Middlebrook $7 \mathrm{H} 10$ medium at $37^{\circ} \mathrm{C}$ for 6 weeks, autoclaved $\left(120^{\circ} \mathrm{C}\right.$ for $30 \mathrm{~min}$ ), washed twice with distilled water, and lyophilized.

Sample preparation for $G C$. A portion (5 mg dry wt) of each strain was heated in $1 \mathrm{ml} 30 \%(\mathrm{w} / \mathrm{v})$ methanolic $\mathrm{KOH}$ at $80^{\circ} \mathrm{C}$ for $30 \mathrm{~min}$ in glass test-tubes fitted with Teflon-lined screw-caps. After cooling, the alkaline hydrolysates were extracted with $2 \mathrm{ml}$ hexane. The hexane phase was used for both GC and GC-MS analysis of alcohols (see below).

A separate portion ( $5 \mathrm{mg}$ dry $w \mathrm{t}$ ) of one of the mycobacterial strains was mixed with $1 \mathrm{ml} 1 \mathrm{M}$-methanolic $\mathrm{HCl}$ and heated at $80^{\circ} \mathrm{C}$ for 30 min (Jimenez \& Larsson, 1986). After cooling, the reaction mixture was shaken with $2 \mathrm{ml}$ hexane. The hexane phase was separated and used for combined GC analysis of fatty acids and fatty alcohols.

Gas chromatography. A Varian model 3500 gas chromatograph equipped with a flame-ionization detector and a split/splitless injector (working in the splitless mode) was used. Analyses were performed on a fused-silica capillary column $(25 \mathrm{~m} / 0.25 \mathrm{~mm}$ i.d. $)$ coated with crosslinked OV-1. Nitrogen, at a flow-rate of $2.5 \mathrm{ml} \mathrm{min}-1$, was used as carrier gas. Both the injector and the detector were held at $280^{\circ} \mathrm{C}$, and the column temperature was programmed from 140 to $280^{\circ} \mathrm{C}$, at a rate of $8{ }^{\circ} \mathrm{C} \mathrm{min}^{-1}$.

Sample preparation for $G C-M S$. The preparations used for GC analysis of alcohols (see above) were also studied by GC-MS. The alcohols were analysed both underivatized and as trimethylsilyl (TMS), methyl ether (METH) or pentafluorobenzoyl (PFBO) derivatives prepared from the hexane extracts (after evaporation to dryness) according to the following procedures. 

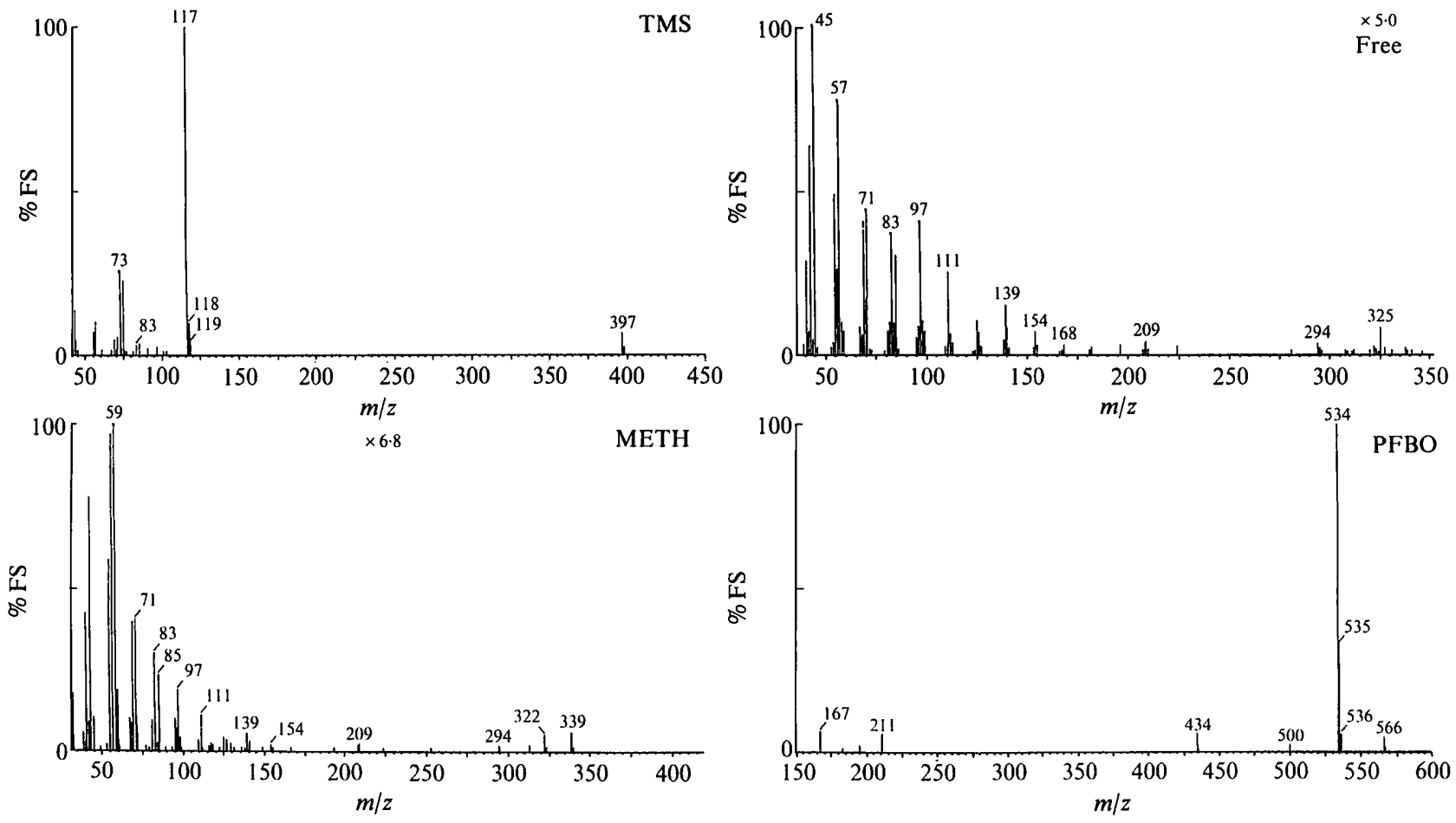

Fig. 1. Mass spectra of one of the two branched-chain secondary alcohols detected (peak no. 7, see Fig. 2). The alcohol was analysed in the EI mode underivatized and as TMS- and METH derivatives and in the NICI mode as a PFBO derivative. \%FS, percentage fullscale deflection.

TMS derivatives were formed by adding BSTFA $(50 \mu \mathrm{l})$ and pyridine $(10 \mu \mathrm{l})$ to the sample and then heating at $80^{\circ} \mathrm{C}$ for $15 \mathrm{~min}$. After cooling to room temperature, pyridine was removed from the reaction mixture with a stream of nitrogen, and hexane was added as final solvent.

METH derivatives were formed by mixing the sample with $60 \mathrm{mg}$ silver oxide and refluxing in $2 \mathrm{ml}$ methyl iodide for $5 \mathrm{~h}$. The reaction mixture was then filtered and the filtrate was collected, dried under a stream of nitrogen, and dissolved in hexane.

PFBO derivatives were prepared by mixing the sample with $35 \%$ (v/v) PFBO-Cl $(40 \mu \mathrm{l})$ and $2 \%(\mathrm{v} / \mathrm{v})$ triethylamine $(20 \mu \mathrm{l})$ (both in acetonitrile) and heating at $80^{\circ} \mathrm{C}$ for $30 \mathrm{~min}$. After cooling to room temperature, hexane $(1 \mathrm{ml})$ and water $(1 \mathrm{ml})$ were added and the mixture was shaken. The organic phase was collected, evaporated and dissolved in hexane.

Gas chromatography-mass spectrometry. A VG Trio-1 S GC-MS system connected to a Hewlett-Packard model 5890 gas chromatograph was used. The gas chromatograph was equipped with a fused-silica capillary column $(25 \mathrm{~m} / 0 \cdot 25 \mathrm{~mm}$ i.d.) containing cross-linked OV-I as the stationary phase. Splitless injections were made using a HewlettPackard model 7673 autosampler. Helium, at an inlet pressure of 7 p.s.i., was used as the carrier gas. The temperature of the column was programmed to rise at $20^{\circ} \mathrm{C} \mathrm{min}-1$ from 120 to $260^{\circ} \mathrm{C}$; both the injector and the interface (between GC and MS) were kept at $260^{\circ} \mathrm{C}$. The ionization was performed at $70 \mathrm{eV}$. The ion-source temperature was $200^{\circ} \mathrm{C}$ in the electron impact mode and $150^{\circ} \mathrm{C}$ in the negative ionchemical ionization (NICI) mode (utilizing methane as reagent gas).

\section{Results}

\section{Mass spectra}

Altogether nine secondary alcohols were detected, and seven of these were identified as 2-octadecanol, 2- nonadecanol, 2-eicosanol, 2-heneicosanol, 2-docosanol, 2-tricosanol and 2-tetracosanol. The electron impact (EI) mass spectra of the underivatized alcohols exhibited fragmentations at $m / z 45, m / z(M-1), m / z(M-15), m / z$ $(M-18)$ and $m / z(M-46)$. The mass spectra of the TMS-derivatized alcohols revealed $\mathrm{m} / z 117\left[\mathrm{CH}_{3} \mathrm{CHO}\right.$ $\mathrm{Si}\left(\mathrm{CH}_{3}\right)_{3}$ ] as the most abundant ion produced, and prominent ions of $\mathrm{m} / z \quad 73 \quad\left[\left(\mathrm{CH}_{3}\right)_{3} \mathrm{Si}\right], \quad m / z \quad 75$ $\left[\left(\mathrm{CH}_{3}\right)_{2} \mathrm{Si}=\mathrm{OH}\right]$ and the molecular-specific ion of $\mathrm{m} / \mathrm{z}$ $(M-15)$ were also observed. The spectra of the METH derivatives showed $m / z(M-15), m / z(M-32)$ and $m / z$ $(M-60)$ as the specific fragments, whereas the molecular ion $(M)$ was the major fragment in the NICI mass spectra of the PFBO-derivatized alcohols.

The remaining two alcohols eluted just after 2eicosanol and 2-docosanol. These alcohols exhibited, respectively, the same mass spectra as 2-heneicosanol and 2-tricosanol, both when analysed underivatized and when analysed as TMS-, PFBO- and METH derivatives (Fig. 1).

\section{Chromatography}

All of the $M$. xenopi strains contained the nine alcohols described. As shown in Fig. 2, the detection of alcohols was facilitated by the use of the selective extraction procedure. In comparison, combined fatty acid and alcohol analysis revealed only 2-eicosanol, 2-docosanol and branched-2-tricosanol (see Discussion) as clearly distinguished peaks. 

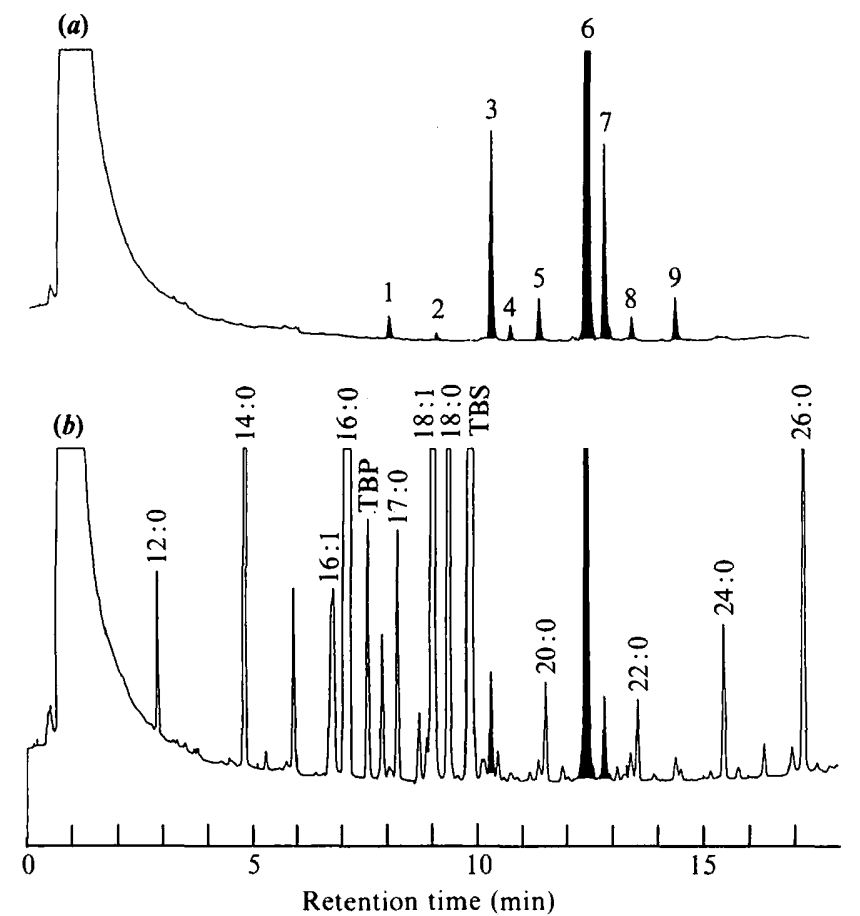

Fig. 2. Gas chromatograms of a strain of $M$. xenopi, obtained after selective extraction of alcohols $(a)$ and by combined fatty acid and alcohol analysis $(b)$. The fatty acids were derivatized to methyl esters whereas the alcohols were underivatized. (a) 1, 2-octadecanol; 2, 2nonadecanol ; 3, 2-eicosanol ; 4, branched-2-heneicosanol ; 5, 2-heneicosanol; 6, 2-docosanol ; 7, branched-2-tricosanol ; 8, 2-tricosanol; 9, 2tetracosanol. (b) TBP, tuberculopalmitate; TBS, tuberculostearate; numbers of carbon atoms and double bonds are indicated using standard nomenclature.

\section{Discussion}

We recently reported that hexane extraction of alkaline hydrolysates followed by GC-MS analysis can be successfully applied to the detection of mycobacterial secondary alcohols: using this procedure, 2-nonadecanol, as well as the previously reported 2-octadecanol and 2-eicosanol, was detected in strains of the $M$. avium complex (Alugupalli \& Larsson, 1992). In the present study on $M$. xenopi, we detected not only 2-eicosanol and 2-docosanol, which have previously been found in $M$. xenopi (Larsson et al., 1989; Luquin et al., 1989), but also 2-octadecanol, 2-nonadecanol, 2-heneicosanol, 2-tricosanol and 2-tetracosanol; the identities of the alcohols were ascertained by GC-MS. Thus, the EI mass spectra of the TMS-derivatized alcohols exhibited abundant ions of $m / z 117$ (breakdown of $\mathrm{C}_{2}-\mathrm{C}_{3}$ ) and $m / z(M-15$ ), whereas the NICI spectra of the PFBO-derivatized alcohols exhibited the molecular ion as the major fragment (Alugupalli \& Larsson, 1992). The EI spectra of the free alcohols revealed fragments at $\mathrm{m} / \mathrm{z} 45, \mathrm{~m} / \mathrm{z}$ $(M-15)$ and $m / z(M-18)$, all characteristic of secondary alcohols (Luquin et al., 1989).
Also detected in all strains were two compounds that eluted just after 2-eicosanol and 2-docosanol (Fig. 2, peaks 4 and 7). When analysed underivatized, both of the compounds showed an abundant peak at $m / z 45$, indicative of a secondary free alcohol. The EI spectra of their TMS derivatives as well as the NICI spectra of their PFBO derivatives were identical with those of 2heneicosanol and 2-tricosanol, respectively. To investigate whether the compounds were iso- or anteiso alcohols, METH derivatives were prepared. This was done since it is known that METH derivatives of straight-chain, iso and anteiso fatty alcohols produce different EI mass spectra: the characteristic fragments of straight-chain alcohols are $m / z(M-60), m / z(M-32)$, and $m / z(M-15)$; those of iso alcohols are $m / z(M-88)$, $m / z(M-60), m / z(M-32)$ and $m / z(M-15)$; and those of anteiso alcohols are $m / z(M-89), m / z(M-61), m / z$ $(M-32)$ and $m / z(M-15)$ (Karlsson et al., 1973). However, the mass spectra of the two METH-derivatized alcohols were found not to differ from those of METH-derivatized 2-heneicosanol and 2-tricosanol: all exhibited major ions at $m / z(M-15), m / z(M-32)$ and $m / z(M-60)$ (Fig. 1). Considering these data, as well as the retention time characteristics of the two compounds, we have drawn the conclusion that they are branchedchain (but not iso or anteiso) secondary alcohols, one with 21 carbon atoms and the other with 23 .

The method described is useful for the determination of microbial fatty alcohols. It remains to be investigated whether a detailed study on the distribution of secondary alcohols in Mycobacterium could provide further information applicable in species identification or chemotaxonomy.

This work received financial support from the Swedish National Association against Heart and Chest Diseases and the Faculty of Medicine, University of Lund, Sweden. We are thankful to Dr Asim Ray, Organic Chemistry 2, Chemical Centre, University of Lund, for valuable discussions.

\section{References}

Alugupalli, S., Mielniczuk, Z. \& Larsson, L. (1992). Gas chromatography-mass spectrometry methods for analysis of secondary alcohols present in the Mycobacterium avium complex. Journal of Microbiological Methods 15, 229-240.

Jantzen, E., TANGEN, T. \& ENG, J. (1989). Gas chromatography of mycobacterial fatty acids and alcohols : diagnostic applications. Acta Pathologica Microbiologica et Immunologica Scandinavica 97, 10371045.

Jimenez, J. \& LARSSON, L. (1986). Heating cells in acid methanol for $30 \mathrm{~min}$ without freeze-drying provides adequate yields of fatty acids and alcohols for gas chromatographic characterization of mycobacteria. Journal of Clinical Microbiology 24, 844-845.

Karlsson, K. A., Samuelsson, B. E. \& Steen, G. O. (1973). Improved 
identification of monomethyl paraffin chain branching (close to the methyl end) of long-chain compounds by gas chromatography and mass spectrometry. Chemistry and Physics of Lipids 11, 17-38.

Larsson, L., Jimenez, J., Valero-Guillén, P. L., Martín-Luengo, F. \& KuBIN, M. (1989). Establishment of 2-docosanol as a cellular marker compound in the identification of Mycobacterium xenopi. Journal of Clinical Microbiology 27, 2388-2390.

LuQuin, M., LoPEZ, F. \& Ausina, V. (1989). Capillary gas chromatographic analysis of mycolic acid cleavage products, cellular fatty acids and alcohols of M. xenopi. Journal of Clinical Microbiology 27, 1403-1406.

Luquin, M., ausina, V., Lopez Calahorra, F., Belda, F., Garcia Barcelo, M., Celma, C. \& Prats, G. (1991). Evaluation of practical chromatographic procedures for identification of clinical isolates of mycobacteria. Journal of Clinical Microbiology 29, 120-130.

MinniKin, D. E. (1982). Lipids, complex lipids, their chemistry, biosynthesis and roles. In The Biology of Mycobacteria, vol. 1, pp. 95 184. Edited by C. Ratledge \& J. L. Stanford. London: Academic Press.

Minnikin, D. E., Minnikin, S. M., Parlett, J. H., Goodfellow, M. \& MAGNusson, M. (1985). Mycolic acid patterns of some species of Mycobacterium. Archives of Microbiology 139, 225-231.

Toriyama, S., Imaizumi, S., Tomiyasu, I., Masui, M. \& Yano, I. (1982). Incorporation of ${ }^{18} \mathrm{O}$ into long-chain, secondary alcohols derived from ester mycolic acids in Mycobacterium phlei. Biochimica et Biophysica Acta 712, 427-429. 